\section{Mujeres Mapuce entretejiendo \\ conocimientos y memorias de resistencia. Notas de investigación}

- Graciela Alonso, Eva Lincan y Anabella Paz

\begin{abstract}
Resumen
El artículo reflexiona sobre categorías que entendemos densifican el abordaje de la investigación en clave intercultural en el trabajo con mujeres mapuce. En ese sentido, las categorías intersección, fusión, memoria y ancestralidad nos permiten pensar que los procesos interculturales, dentro y fuera de los espacios mapuce, proyectan sus contenidos y metodologías sobre la base de la resignificación y transmisión de pedagogías ancestrales en clave generacional y de resistencia a un conjunto de opresiones.
\end{abstract}

\section{Mapuce women interweaving knowledges and memories of resis- tance. Research notes}

\begin{abstract}
This article reflects on categories that according to our understanding densify the approach of intercultural investigation when working with mapuce women. In this respect, categories such as intersection, fusion, memory and ancestrality enable us to consider that intercultural processes, both inside and outside mapuce sites, project their contents and methodologies on the basis of resignification and transmission of ancestral pedagogies in generational terms and in terms of mapuce resistance to a set of oppressions.
\end{abstract}

\section{Introducción}

Brevemente referiremos a algunas cuestiones de contexto. En las últimas décadas el Estado, en convivencia con intereses empresariales, profundizó los avances neoextractivistas en Patagonia, reinstalando la idea de "progreso" ligada a la depredación de los

\section{Palabras clave:}

Pedagogía, ancestralidad, resistencia, interseccionalidad.

Keywords:

Pedagogy, ancestrality, resistance, intersectionality. 
1. Espíritu del este. Las palabras en Mapuzugun serán traducidas a pie de página, la primera vez que aparezcan en el artículo. Se utiliza el grafemario Raguileo.

2. Este escrito fue iniciado por una de las integrantes mapuce del equipo de investigación en su trabajo de tesis de licenciatura y luego compartido y enriquecido con los aportes del Proyecto de Investigación por el cual estamos transitando: "Resistencias territoriales en clave de género: mujeres mapuce tejiendo estrategias comunitarias frente a las múltiples violencias en la actual fase del capital".

3. Plantas, hierbas para uso medicinal.

4. Conocimiento.

5. Usamos la expresión contextos de urbanidad para diferenciar el asentamiento de la comunidad en un ámbito que hoy no es rural. Lo hacemos a sabiendas de que el

Pueblo Mapuce no acuerda con la división urbano/rural para caracterizar a las comunidades. Esa lógica es utilizada por el Estado para intentar no reconocer a las comunidades que en sus desplazamientos han quedado insertas en las actuales ciudades. Las entrevistas abiertas se realizaron en la ciudad de El Bolsón que tiene particularidades que hacen difusa la división entre lo rural y lo urbano. La observación participante se realizó y se realiza en la ciudad de Neuquén, en un barrio en el que se concentró gran parte de la población mapuce llegada de las comunidades emplazadas en Junín de los Andes. bienes comunes, fundamentalmente a través del fracking y la actividad minera. Focalizar en la explotación de estos recursos se ha convertido, además, en una estrategia política que coloca a nuestros pueblos originarios en una situación de fragilidad/resistencia, ya que invade el espacio territorial en todas sus dimensiones.

Este avance es entendido por las mujeres mapuce con las que venimos conversando en las comunidades Puel Pvjv, ${ }^{1}$ Campo Maripe (ambas en Neuquén) y en El Bolsón, como una continuación del genocidio iniciado con la conquista del "desierto" y profundizado mediante diversos dispositivos estatales. Estas estrategias impusieron violencia real y simbólica como una forma sistemática de relación con los pueblos originarios. El genocidio se reactualiza y se profundiza ante la continuidad de los despojos territoriales y las diversas formas de represión, que vulneran derechos fundamentales a la salud, a la alimentación y a la vida misma. La contaminación se convierte entonces en un dispositivo que invade cuerpos, territorios, conocimientos. Claras muestras de la realidad que atraviesan hoy las comunidades del pueblo mapuce con las que interactuamos en contextos urbanos o rurales.

El escrito tiene como principal objetivo recuperar parte de las tareas que han llevado y llevan adelante mujeres mapuce que habitan territorio originario en la Patagonia urbana. Mujeres que, con su diario quehacer, condicionadas fuertemente por las representaciones sociales y culturales impuestas por la colonización patriarcal, han logrado sostener los hilos que tejen y nutren la historia del pueblo mapuce. ${ }^{2}$

Buscamos hacer visible las tareas de estas mujeres, y con ello el mundo simbólico que encierran sus conocimientos, en el tejido de una prenda, en el reconocimiento de un lawen, ${ }^{3}$ en la transmisión de su kimvn, ${ }^{4}$ generando en estos actos, procesos que permitieron resistir y reexistir en un mundo que les dio un lugar de subalternidad. Esta subalternidad se concentró en sus cuerpos. Parafraseando a Millaleo (2016) los cuerpos femeninos indígenas son ubicados en el imaginario social como "cuerpos de servicios".

Pretendemos reflexionar sobre algunos de estos actos de resistencia, focalizando en la transmisión de conocimientos donde la memoria juega un papel preponderante. Los interrogantes que nos fuimos formulando guían la búsqueda de respuestas, provisorias, situadas:

» ¿Cómo se comprometen/enlazan el cuerpo, la memoria, la historia de las mujeres del pueblo mapuce en la transmisión de conocimientos?

» ¿Cómo son construidos/configurados/reconfigurados estos conocimientos desde las jóvenes generaciones en espacios urbanos?

El trabajo de campo fue realizado a través de dos estrategias específicas: la entrevista etnográfica y la observación participante. Las entrevistas se hicieron a mujeres mapuce en contexto de urbanidad ${ }^{5}$ que no han vivido en comunidad, pero que lograron aprender, sostener y transmitir conocimientos propios de la cosmovisión, a través de actividades específicas que llevan adelante en sus vidas cotidianas y en el desempeño de sus profesiones como enfermera, tejedora y ama de casa. Las entrevistas se desarrollaron principalmente entre 2015 y 2017; en todos los casos, fueron como mínimo tres encuentros (entre sesenta y noventa minutos) con cada entrevistada en forma individual. La observación participante corresponde al taller de Arte Medicina. Se produjeron cinco registros en el transcurso del año 2018. El trabajo se retomará en el mes de mayo de 2019. De los encuentros participan sostenidamente seis mujeres mapuce.

Con ambas estrategias se busca reflexionar respecto a cómo, en los diferentes espacios se va construyendo una pedagogía de resistencia, de la sanación, una pedagogía que interpretamos como pedagogía de la ancestralidad. Esta pedagogía se entrama, por un 
lado, en los llamados ámbitos domésticos -volveremos sobre esta denominación-y por el otro, en ámbitos educativos comunitarios urbanos o interculturales. La ancestralidad acompaña la construcción de formas de reexistencia ${ }^{6}$ tanto personal como colectiva.

El artículo reflexiona sobre categorías que entendemos densifican el abordaje de la investigación en clave intercultural en el trabajo con mujeres mapuce. En ese sentido las categorías intersección, fusión y memoria nos permiten pensar que los procesos interculturales, dentro y fuera de los espacios mapuce, proyectan sus contenidos y metodologías sobre la base de la resignificación y transmisión de pedagogías ancestrales en clave generacional y de resistencia mapuce a un conjunto de opresiones.

\section{Notas de investigación}

La dimensión intercultural atraviesa y anuda nuestro trabajo en varios puntos. Uno de ellos es la relación entre el trabajo académico y el militante. Somos docentes, estudiantes, graduadxs universitarias y algunas pertenecientes al pueblo mapuce. El nuestro es un largo proceso y recorrido de más de veinte años. ${ }^{7}$ Las coyunturas políticas y problematizaciones teóricas en el campo, nos han permitido tomar la problemática intercultural en diferentes momentos, dejarla en otros y retomarla, desde varias y situadas estrategias, en espacios y tiempos particulares.

Consideramos como un aporte fundamental en este/nuestro recorrido teórico epistémico (político), el diálogo con teorías feministas y, posteriormente, con las descoloniales. El feminismo negro, la concepción de interseccionalidad y el debate acerca de las luchas de mujeres indígenas, y los feminismos comunitarios y territoriales, nos fueron nutriendo para la extensión crítica y replanteo de experiencias de investigación intercultural.

Con relación a la interculturalidad la pensamos desde fines de los años noventa enfáticamente crítica, desde una estrategia de desconstrucción y descolonización, no reducida al estudio de la diversidad étnico-cultural, sino apuntando a la refundación estructural y plurinacional de los estados latinoamericanos, tal como lo fuimos aprendiendo y construyendo junto a organizaciones mapuce.

Las propuestas interculturales emergen de saberes "otros" enraizados en espacios de disidencia y de construcción de sociedades "otras".

Fuimos recorriendo un camino de análisis acerca del cuerpo y su contenido político; así, la consigna "lo personal [también] es [o puede ser] político", creada y difundida por el movimiento feminista, está siendo en nuestras propuestas, registrada, extendida y resignificada para lograr que sea inclusiva de las voces de mujeres jóvenes mapuce. Nos referimos por ejemplo al debate acerca del aborto que están instalando jóvenes del Lof Puel Pvjv. ${ }^{8}$ Esta interpelación provoca también experiencias concretas de resistencia de las mujeres indígenas, la presencia y proyección como pueblo originario.

Los encuentros generacionales y diálogos interculturales nos facilitan entrever la construcción de una pedagogía de/desde la ancestralidad, que no siempre es visibilizada en tanto tal pero que se va configurando en ese espacio interseccional. Este espacio vacío/vaciado no muestra solo la ausencia de sujetxs, sino también los contenidos que han sido desplazados.

Para las ciencias sociales, el concepto o la perspectiva interseccional impele a análisis complejos sobre las diferentes formas de opresión y las maneras en que la "herida colonial" se encarna en cuerpos situados en procesos particulares y locales. Es necesario
6. Tomamos este concepto de Adolfo Albán Achinte (2012), “Epistemes "otras'. Epistemes Disruptivas”.

7. Agradecemos el acompañamiento de Raúl Díaz de larga trayectoria en el tema y actual asesor de nuestros proyectos de investigación.
8. Comunidad (Lof) Mapuce de la ciudad de Neuquén. 
que la investigación los resitúe para evitar prefigurar o desarrollar una interculturalidad colonizadora. Para el activismo político anticolonial resulta fundamental la lucha antirracista y antipatriarcal en todos los planos.

La categoría intersección nos viene siendo útil para la problematización de temas/ objetos de investigación y también para la construcción de articulaciones varias. El feminismo en Abya Yala está sabiendo construir/construirse desde la visibilidad y valoración de los cruces, las fusiones.

No nos interesa en este espacio debatir la perspectiva de sumas de opresiones versus la de intersección (económica, política e ideológica que se coconstituyen para oprimir diferencialmente a las mujeres), sino ver la posibilidad que tiene la categoría de levantar análisis aplicados, sobre experiencias de resistencias de mujeres indígenas de distintas generaciones que encuentran en el espacio de la memoria un punto de articulación y proyección.

En todo caso, una perspectiva intercultural crítica guiando la investigación, nos ha hecho reflexionar acerca de las invisibilizaciones que provocamos al ser parte, como investigadorxs, de las operaciones de reproducción de los lugares vacíos/vaciados de la intersección; o lo que sería equivalente, el compromiso con el lado oscuro/oculto de la colonialidad de poder y de género (Lugones, 2008).

Como sostiene María Lugones (2008) la separación categorial no solo provoca un problema de "ceguera epistemológica", sino que provoca también la distorsión de los nudos de poder que existen en la intersección. En consecuencia, se dejan fuera las sobredeterminaciones múltiples entre las categorías. En la intersección entre mujer y negro hay una ausencia donde debería estar la mujer negra precisamente porque ni mujer ni negro la incluyen. La intersección muestra un vacío. Por eso, una vez que la interseccionalidad nos muestra lo que se pierde, nos queda por delante la tarea de reconceptualizar la lógica de la intersección para, de ese modo, evitar la separabilidad de las categorías dadas y el pensamiento categorial.

... Esto implica que el término mujer en sí, sin especificación de la fusión no tiene sentido o tiene un sentido racista, ya que la lógica categorial históricamente ha seleccionado solamente el grupo dominante, las mujeres burguesas, blancas heterosexuales y por lo tanto ha escondido la brutalización, el abuso, la deshumanización que la colonialidad de género implica. (Lugones, 2008, p.25)

En la cita de Lugones, entendemos violencia contra las mujeres en sentido amplio, tanto a nivel epistemológico y cultural como a nivel de los desplazamientos territoriales. Intentando comprender la complejidad que se teje en la resistencia cuando se visibilizan los cruces y traslaciones entre el afuera y el adentro pero también dentro de los propios procesos políticos de las comunidades, por ejemplo, interpelaciones desde posiciones de género y generación.

Consideramos que una labor de las construcciones pedagógicas interculturales interseccionadas, es reponer aquello del orden de lo desplazado. Claramente no suponiendo que existe una restitución como copia original, sino una restitución que se hace cargo de la lucha que significa la memoria, que se abre paso, como puede $-\mathrm{y}$ a veces como quiere-; poniéndole el cuerpo a los avatares de las interpelaciones, del conjunto social. Por ello, proponemos revisar las propias categorías de conocimiento volviéndolas sensibles a los debates, desacuerdos y disputas por la visibilidad de las resistencias. 
Sostiene Lugones que:

... la resistencia proviene de la participación alternativa de una comunidad que es anticapitalista, antirracista, antisexista, una comunidad que tiene un sistema de valores distintos. La resistencia de la mujer negra para confrontar esta dominación se da en términos interseccionales, sin embargo, sus experiencias son distintas y ellas son distintas unas de las otras en cómo responden. (Lugones citada por Bidaseca, 2014, p.958)

Tomamos esta cita porque venimos observando que las mujeres jóvenes mapuce con las que estamos en relación, construyen comunidad también desde una perspectiva crítica al sexismo, revisitando historias individuales y colectivas en este andar.

Del trabajo de Lugones, el concepto que nos interesa retomar en este artículo es el de fusión, quizás menos recuperado en su obra. En un interesante trabajo Karina Bidaseca (2014), levanta de Lugones la importancia del concepto de fusión.

... en mi caso yo pienso en la posibilidad de actividad, de una agencia tal vez mínima pero importante solamente si la persona es impura, es decir, no es alguien que valora la homogeneidad ni la pureza en sí misma ni lo que construye lo social, sino es alguien donde raza y género están fusionadas, pero en esa fusión ellas siguen siendo oprimidas: hay una correlación resistiendo. [...] Para mí la mujer es social; ese sujeto cuando está siendo oprimido resiste, no hay (un) resistiendo sin ser siendo oprimida y viceversa, yo lo pongo en el gerundio. (Lugones citada por Bidaseca, 2014, p.959)

En este gerundio es en el que nos encontramos con las mujeres mapuce, sabiendo de las desigualdades que nos atraviesan.

\section{Pedagogía mapuce en resistencia ${ }^{9}$}

Por ahí se daba la charla, por ahí estábamos en una tarde y la abuela... yo recuerdo que por ahí la abuela estaba en la ramada que tenían ellos [...] que era para trabajar. La abuela yo me acuerdo que tenía ella unos tachos... por ahí preguntaba por curiosidad ¿qué es abuela? Y bueno, estoy haciendo tal cosa y ver que estaban ahí los alambres, las madejas... de eso me acuerdo yo, de los colores, los distintos colores, de eso me acuerdo que ella me explicaba. (S. A., entrevista, 2018).

La repetición de estas diversas formas de violencia fragmentó a lo largo del tiempo, el sostén que brinda un espacio territorial y una cosmovisión compartida, impactando fundamentalmente en la transmisión de conocimientos, debilitando el Nor Kimvn, ${ }^{10}$ pilar que sostenía y sostiene la educación autónoma mapuce. En la concepción de educación del pueblo mapuce, el kimvn debe ser transmitido en forma oral en diferentes espacios y momentos de la vida comunitaria. Se realiza a través del mapuzungun. ${ }^{11}$ Es central la relación que se establece entre todos los elementos, donde la persona es un elemento más del waj mapu, ${ }^{12}$ regida por principios y valores que regulan su pertenencia territorial y familiar, otorgando una identidad particular a cada $c e .{ }^{13}$ Estos conocimientos se transmiten de generación en generación, ordenando la relación entre las diversas vidas del waj mapu.

Esta concepción piensa la transmisión del conocimiento de manera circular, otorgando un significado importante a la idea de igualdad y complementariedad. Las mujeres son poseedoras, a través de transmisiones generacionales, de pewma ${ }^{14} \mathrm{y}$ de conocimientos ancestrales que les permite llevar adelante determinados roles, asumiendo lugares de autoridad.
9. "Comunicación personal" referirá a entrevistas hechas por el equipo de investigación. Las siglas corresponden a las personas entrevistadas. "Registro de observación" referirá a notas/registro de un conjunto de observaciones.

10. Sistema de conocimiento.

11. Idioma mapuce. En este artículo se utiliza el grafemario Raguileo.

12. Territorio.

13. Persona.

14. Sueños. 
Los testimonios que siguen muestran cómo la concepción ancestral de educación, sostenida fundamentalmente por las y los ancianos, son una forma de transmisión y reafirmación de identidad, aún en contextos de urbanidad. Lo particular es que, a raíz de desplazamientos forzosos/forzados son las abuelas quienes buscan espacios de transmisión que involucran la memoria y comprometen el cuerpo. Lo hacen a través de relatos y vivencias que se transmutan en el hacer y en el gvlam: ${ }^{15}$

... Y ahí yo empecé a soñar, a tener pewma con plantas, sobre todo plantas, [...] Y después de haber hecho todo el proceso, [escuchar a] la anciana, a la kushe decir: -Ah! Yo había soñado que uste podía hacer eso. (G. P., comunicación personal, 2015)

Así nos criamos, muy ligados a los abuelos [...] Aprender a leer cosas... del cielo, de cómo estaba, cuando salía la luna, cómo eran las nubes... bueno, un montón de cosas... son cosas que naturalmente te van transmitiendo, no es que te van diciendo bueno, mirá esto, mira lo otro.... No, sino que se hacía... (G. P., entrevista, 2017)

... el tema del telar... lo aprendí de mi abuela y el tejido, el hilado, el telar, todos esos conocimientos lo aprendí de ellas, de mi mamá y de mi abuela. [...] yo la miraba cuando ella armaba [el telar], pero nunca le dije "a ver abuela" ni tampoco ella me explico "así se hace", no, yo solamente me sentaba ahí, tranquilita, y ahí miraba, observaba nomás... (S. A., entrevista, 2018)

... moler el trigo, moler arvejas... y eso lo aprendí de mi abuela. Ella no tenía molinillo, tenía piedra, [...] he usado piedra alguna vez y es impresionante porque son piedras que han usado nuestras tatarabuelas, entonces vos, arrodillarte, agarrar esas piedras, moler donde molió la abuela de tu abuela... es muy fuerte. (G. P., entrevista, 2018)

Estos testimonios remiten a una transmisión y aprendizaje recibido y resignificado en espacios como la casa. Es factible pensar estos espacios domésticos como formas de resistencias que resguardaron y fortalecieron las mujeres. Los conocimientos transmitidos de abuelas a nietas en la intimidad de una ramada, por ejemplo, se convirtieron también en formas de comunicación personal, femenina. Tal vez las tramas de un tejido, el uso del lawen, fueron la forma que encontraron las mujeres de dar su voz. Por eso pensamos, como advierte Segato (2016), que lo doméstico no siempre es un espacio carente de politicidad. Si lo comunitario lleva en sí el germen de existencia como pueblo nos vemos impelidas a considerar lo que se entiende por doméstico de otros modos, desafinado la oposición binaria público/privado.

Nos parece necesario profundizar la investigación intercultural volviéndola crítica respecto del tratamiento de algunos conceptos, tal como el del espacio doméstico y las tareas de cuidado, evitando trasladar sin mediaciones la negatividad implícita que conlleva lo no-público y haciendo visibles formas de transmisión, pedagogías de la ancestralidad, que ejecutadas por mujeres en ámbitos no tan visibles, entretejen de todos modos, y a veces decisivamente, la politicidad y la resistencia de las mujeres mapuce.

Otro espacio que relevamos, en el cual las mujeres dan su voz, es en el taller de arte/ medicina que se sostiene en el Lof Puel Pvjv de la ciudad de Neuquén. En este espacio se plasman pinturas en tela o papel. Las mujeres del Lof pintan sus pewma, ya que, a través de ellos, las fuerzas ancestrales transmiten conocimientos. Conciben el arte medicina como instancias de sanación a través de la memoria ancestral. Sostienen que la concepción que abordan, rompe con las lógicas hegemónicas, académicas del campo del arte ya que lo conciben como medicina en sí misma y no como elemento terapéutico complementario. 
El arte medicina es un arte que es netamente espiritual, no visto el arte como una terapia porque comúnmente escuchamos así en la sociedad, en el mismo sistema que se aconseja hacer determinadas técnicas de pinturas o actividades artísticas como a nivel terapéutico. Y se dice que es una terapia que ayuda, es cierto que ayuda y la lógica terapéutica es muy buena, pero cuando se habla de arte medicina no se refiere a que el arte medicina sea considerado como una terapia. Sino que el arte por sí mismo es medicina, por ejemplo, en el arte que nosotros estamos compartiendo en la comunidad es un arte que es medicina porque es un arte que está trayendo a la realidad, en la tela o en lo que estén pintando, están trayendo toda la cosmovisión que cada lamgen ${ }^{16}$ tiene... (M. P., entrevista, 2018)

Todas las personas tenemos algo que sanar, a través del arte medicina podemos volcar nuestra memoria ancestral que se manifiesta en los sueños, es ahí donde nos comunicamos con la diversidad de vidas y es ahí donde se va produciendo un proceso de sanación. Y es real porque acá muchas de nosotras nos hemos curado de dolencias y enfermedades físicas, así como te lo digo y eso nos fortalece. (E. P., registro observación, 2018)

El arte, anclado en el conocimiento ancestral en el cual se refleja el conocimiento mapuce, puede ser comprendido como prácticas de fortalecimiento inscriptos en las estrategias de recuperación de la memoria en contextos de urbanidad. Desde el taller se definió, comunitariamente, que el arte medicina consiste en la curación de la comunidad y de las personas. "Es un espacio para curar, para crear recuperando la cosmovisión, el conocimiento propio de los territorios y pu newen, ${ }^{17}$ los sueños y las luchas que nos atraviesan" (C. V., registro observación, 2018).

Las curaciones ancestrales se reconstruyen, se alían con otras formas de conocer y aprender, y se plasman hoy en espacios donde la educación autónoma circunscribe a la educación intercultural, a la vez que conformando estrategias de fortalecimiento interno; se abren a espacios interculturales como parte de las luchas y el protagonismo/ disputa de/por otras formas de conocimiento.

En estos sentidos interpretamos que hay una profunda trama de saberes desde los cuales se fortalece la identidad comunitaria, buscando reparaciones profundas a una historia que se impuso.

\section{La memoria como pedagogía de resistencia}

Como venimos planteando, las diferentes formas de violencia condicionaron la posibilidad de dar continuidad a formas de vida. Situamos esto en lo que Ana Ramos (2017) denomina la aplicación sostenida por parte del estado nacional, de "fórmulas de borramiento" que han producido silencios y o interrupciones en las transmisiones. Movimientos que, a través de los desplazamientos forzados, generaron procesos de sometimiento en las trayectorias de las mujeres mapuce. En este movimiento fue donde las familias en general y las mujeres en particular, sufrieron profundas modificaciones en sus formas de vida, ya que se las introdujo no solo en otras maneras de ver el mundo y relacionarse con él, sino también en cambios al interior de los grupos familiares y de la comunidad misma. Tejendera de memorias:
16. Hermana.

17. Pu se utilizar para hacer plural las palabras. Newen se traduce como espíritu. 
Mi mamá también empezó a tejer ya de grande, pero ella empezó ya con todo, dibujo, todo... como si estuviera en algún lado de su cabeza y como que vuelve ¿no?... (G. P., comunicación personal, 2015)

Yo el tema del laboreo [tejido] lo fui aprendiendo mirando, así, viendo cómo... inventando digamos, lo aprendí de algún lugar... (S. A., entrevista, 2018)

Ramos, Crespo y Tozzini toman el concepto de memorias subterráneas o clandestinas de Pollak para dar cuenta que, en sujetos y comunidades atravesadas históricamente por el sufrimiento y la violencia, la memoria es reapropiada y “...transmitida al interior del ámbito familiar o de asociaciones, redes de sociabilidad afectiva y/o política” (2016, p.24). De este modo, y en coincidencia con las autoras citadas, consideramos que es factible sobrellevar las marcas del dolor y generar en los espacios cotidianos novedosas formas de recuperación y retransmisión de tramas culturales en un movimiento pedagógico que se inaugura en los nuevos espacios, recreando el kimvn, buscando, además, descolonizarlo de los sentidos dados hegemónicamente.

Las ancianas mujeres mapuce han practicado formas de transmisión de conocimientos con las nuevas generaciones, a través de vivencias corporizadas. Para explicar este punto de vista, recurrimos a Ahmed quien afirma que "El dolor no es simplemente el efecto de una historia de daño: es la vida corporal de esa historia" (2015, p.68). El pueblo mapuce ha sufrido este dolor, de desmembramiento, de prohibiciones. El lenguaje, el mapuzungun como sostén, hilo conductor de una historia común, así como los espacios de socialización comunitarios debieron ser olvidados, negados y disciplinados. En esta represión quedó la posibilidad de enseñar en espacios familiares, generando una pedagogía de la ancestralidad, en la medida que el traspaso del kimvn se llevó adelante a través del hacer. El cuerpo, involucrando procesos de memoria, permitió a las nuevas generaciones recordar y recrear. Por eso interpretamos que esos espacios no se pueden definir como domésticos, en su acepción de privados, ya que hay una prevalencia simbólica y ambiental de lo comunitario.

Los relatos de las lamgen, ponen de manifiesto los procesos de transmisión de conocimiento a través de la relación cuerpo-memoria. Procesos de transmisión donde las miradas, el ir viendo, inventando, sabiendo pertenecen al ámbito de las corporalidades físicas. Al referirse a que el conocimiento fue aprendido en algún lugar se remite a lo que estamos entendiendo por recuperación de la memoria que genera procesos intergeneracionales de conocimiento. Estos movimientos permiten configurar lo que hemos denominado como pedagogías de la ancestralidad. Nos interesa visibilizar los cuerpos de las mujeres como espacios de resistencia-ancestralidad, y como un eje de fusión intergeneracional.

Las nuevas generaciones de mujeres mapuce no están ajenas a procesos de violencia reales y simbólicas instituidas por la sociedad hegemónica y el estado. La mayoría ha sufrido situaciones que condicionan aquellos conocimientos que han logrado ser apropiados por la memoria, disciplinando el cuerpo y la mente, para responder al lugar asignado en el marco de lo considerado "civilizado", incorporando o resistiendo estas representaciones hegemónicas en su subjetividad.

No obstante, dichas representaciones vienen siendo desafiadas. Ana Ramos menciona que las memorias hegemónicas instalan sentido de realidad en la vida cotidiana, pero también "... estas historias pueden ser resignificadas y reutilizadas como proyectos propios...” (2016, p.24). En lo particular, consideramos que las mujeres mapuce están elaborando nuevos sentidos colectivos y contribuyendo a dar continuidad al traspaso de conocimientos, permitiendo por un lado sostener los hilos identitarios que configuran una cosmovisión y por otro, producir conocimientos en nuevos contextos. 
En los procesos en los que nos estamos referenciando, es factible reconocer dos categorías que utiliza Ramos respecto a la memoria: "Memoria conocimiento", aquella que no solamente recuerda, sino que también produce conocimientos y "memoria subjetivación", que articula las experiencias del afuera con las profundidades de nuestro interior. "Conocer es ser capaz de volver a contar historias del mundo, pero desde la percepción de los cambios sutiles del entorno" (2016, p.55).

Yo hoy por hoy doy clases, tengo un grupo de mujeres, que le doy taller y yo les digo como es el proceso, como es el oficio de tejendera... (S. A., entrevista, 2017)

... hacer salud social y comunitaria [...] estuvimos yendo a las comunidades de la meseta en Gastre, estuvimos en Yala Laubat, Lagunita Salada y Blancuntre y también, siempre como revalorizando en esos espacios la medicina mapuce, cuáles plantas usan... Hicimos talleres con la gente, de preparados, talleres de reconocimiento de plantas. (G. P., comunicación personal, 2017)

Entendemos que enseñar a otras y compartir espacios de conocimientos es una manera de transmisión y resignificación de los saberes que fueron aprendidos a partir de la observación y la acción, retenidos en la memoria, puestos en juego, irrumpiendo en espacios hegemónicos y logrando dar una continuidad histórica. Entendemos también estas construcciones y espacios de encuentro como reparadores del dolor social, colectivo, comunitario que ha transitado el pueblo mapuce y en particular las mujeres. Sus intervenciones son una manera de resistir y reexistir a la lógica patriarcal, al proyectar hacia el afuera prácticas que fueron ubicadas (y despolitizadas) en el ámbito de lo privado/doméstico. Consideramos a partir de estas afirmaciones, que es factible encontrar en esta transmisión de conocimientos, prácticas pedagógicas interculturales que desafían la lógica de la colonialidad, haciendo circular una mirada diferente de ver y entender el mundo.

\section{Recapitulando}

Como síntesis provisoria, retomamos las preguntas con las que se inició el escrito, acerca de la manera en que se compromete el cuerpo, la memoria, la historia, en la transmisión de jóvenes mujeres mapuce, conocimientos en espacios urbanos y cómo son apropiados por las nuevas generaciones en procesos de resistencias que implican fusiones. $\mathrm{Al}$ respecto nos parece importante recuperar el lugar donde intersectan la memoria y el cuerpo, pensarlas en movimiento, como procesos que activan y reactivan lazos generacionales, familiares, comunitarios.

Pensamos que en esta pedagogía de la ancestralidad es posible proyectar y fortalecer las luchas de nuestros pueblos, que estas prácticas que parecen aisladas o personales se convierten en política al emerger en espacios públicos, pero también fortaleciendo los procesos de construcción comunitaria.

Procesos que constituyen prácticas pedagógicas en las que el hacer se convierte en modos de resistencia, contribuyendo tanto a sostener la cosmovisión en la vida cotidiana como a resignificarlos en una práctica política que busca fundamentalmente fortalecer la identidad, defender y reivindicar modos de vidas otros, de acuerdo con valores ancestrales presentes en la/nuestra cosmovisión.

La investigación social una vez más se ve desafiada a usar gerundios que permitan visibilizar procesos de resistencia en las opresiones; fusiones en las intersecciones; interculturalidad en los procesos de autonomía y memoria. 


\section{- Referencias bibliográficas}

"Ahmed, S. (2015). La Política Cultural de las Emociones. Ciudad de México, México: UNAM.

"Albán Achinte, A. (2012). Epistemes "otras": ¿epistemes disruptivas? KULA. Antropólogos del Atlántico Sur, (6), 22-34. Recuperado de: http://www.revistakula. com.ar/wp-content/uploads/2014/o2/KULA6_2_ALBAN_ACHINTE.pdf

» Bidaseca, K. (2014). Los pregrinaje de los feminismo de color en el pensamiento de María Lugones. En Estudos Feministas, 22 (3), 953-964. Florianópolis, Brasil. Recueprado de: http://www.scielo.br/pdf/ref/v22n3/14.pdf

"Lincán, E. (2013). Saberes en la constitución de identidad en mujeres mapuce urbana. Ponencia presentada en VI Congreso de Investigación Educativa de la Universidad Nacional del Comahue, Neuquén, Argentina.

» Lugones, M. (2008). Colonialidad y género. En Tabula Rasa, (9), 73-101. Universidad Colegio Mayor de Cundinamarca, Bogotá, Colombia.

» Millaleo Hernández, A. (2016). Ser “nana” en Chile: un imaginario cruzado por género e identidad étnica. En Mujeres y pueblos originarios. Luchas y resistencias hacia la descolonización. Santiago, Chile: Pehuen - CIIR.

»Ramos, A. (2017). Cuando la memoria es un proyecto de restauración: el potencial relacional y oposicional de conectar experiencias. En Bello, A., González, Y., Rubilar, P., Ruiz, O. (eds.), Historias y Memorias. Diálogos desde una perspectiva interdisciplinaria, 32-50. San Pablo, Brasil: Universidad de la Frontera.

» Ramos, A., Crespo, C. Tozzini, M. (2016). Memorias en lucha. Recuerdos y Silencios en contextos de subordinación y alteridad. Rosario, Argentina: Uiversidad Nacional de Rosario.

"Segato, R. (2016). La guerra contra las mujeres. Madrid, España: Traficantes de sueños.

\section{Graciela Beatriz Alonso}

Es magister en Investigación Educativa, Universidad Humanismo Cristiano - Programa Interdisciplinario de Investigación en Educación (PIIE), Santiago de Chile. Profesora en Ciencias de la Educación, Universidad de Morón. Docente Departamento de Política Educativa, Facultad Ciencias de la Educación, Universidad Nacional del Comahue. Investigadora en la Universidad Nacional del Comahue y en el Instituto Patagónico de Estudios en Humanidades y Ciencias Sociales, Neuquén, Argentina. Correo electrónico: gracielafem@gmail.com

\section{Eva Noelia Lincan}

Profesora en Ciencias de la Educación, Facultad de Ciencias de la Educación. Universidad Nacional del Comahue (UNCo), Neuquén, Argentina. Mapucezomo, profesora en Ciencias de la Educación (UNCo). Profesora de Enseñanza Primaria 
(Instituto de Educación Superior 809, Esquel). Profesora Especializada en Insuficientes Mentales (Instituto de Educación Superior 804, Esquel). Docente Jubilada. Correo electrónico: evalincan@yahoo.com.ar

\section{Rita Anabella Paz}

Estudiante del Profesorado en Ciencias de la Educación, Facultad de Ciencias de la Educación, Universidad Nacional del Comahue, Neuquén, Argentina. Correo electrónico: anabellapaz685@hotmail.com. 
